\title{
Gangrenous cholecystitis during hospitalization for SARS-CoV2 infection
}

\author{
Emanuele Asti ${ }^{1} \cdot$ Andrea Lovece $^{1} \cdot$ Luigi Bonavina $^{1}$ (]
}

Received: 12 May 2020 / Accepted: 20 May 2020 / Published online: 26 May 2020

(C) Italian Society of Surgery (SIC) 2020

The clinical scenario of abdominal emergency surgery in patients with suspected Covid-19 infection has been addressed by several investigators after the beginning of the recent pandemia. In such circumstances, priority has been given to security protocols to prevent spreading of infection and provide the highest level of protection to the hospital personnel. In a recent cohort study from China, four patients underwent emergency laparotomy for peritonitis immediately after the collection of oropharyngeal swab samples. Testing for Covid-19 resulted negative and patients had an uneventful postoperative course. The main lesson from this study is that, given the narrow time window in emergency surgery, there is no reason to wait for Covid-19 testing, and protection of medical personnel remains the priority [1].

We wish to highlight and discuss a rather different clinical scenario, that is the case of surgical emergencies that occur during hospitalization for established SARS-CoV-2 infection in patients who are recovering from pneumonia. As of February 2020, Italy became the second epicenter of Covid-19 in the world. Most general hospitals in Lombardy and Milan area, including our 450-bed University Hospital, were converted to Covid facilities to provide intensive-care resources for this patient population. Over the past 2 months, we managed three patients, one female and two males aged 86,72 , and 40 years, respectively, who developed acute abdomen while recovering from Covid-19 pneumonia. Contrast-enhanced abdominal CT scan revealed markedly distended gallbladder and decreased wall enhancement consistent with acute acalcolous cholecystitis (Fig. 1). Emergency laparoscopy confirmed gallbladder gangrene in all, associated with fundic microperforation in the youngest patient, and cholecystectomy was completed without complications and no conversion. To prevent aerosolization, the pneumoperitoneum was set at $9 \mathrm{mmHg}$, use of electrocautery was minimized, and smoke was safely aspirated through a filtered smoke evacuation system.

Acalcolous, gangrenous cholecystitis is an insidious clinical condition which typically occurs in hospitalized, critically-ill, and immunosuppressed patients. At present, the pathogenetic relationship of gangrenous cholecystitis with COVID-19 infection is unknown. Gallbladder vasculitis with fibrinoid necrosis has been described in the context of autoimmune disorders, and has been associated with hydrops [2] Interestingly, a coagulation dysfunction has been recognized as a risk factor associated with ARDS and death in Covid-19 patients [3, 4]. Furthermore, it has been shown that SARSCoV-2 may cause endotheliitis in several organs where the angiotensin-converting enzyme 2 receptor is expressed [5]. It is possible that vessel microthrombosis develops even in the absence of overt disseminated intravascular coagulation [6], and that multiple factors including bile stasis, gallbladder distension, systemic inflammation, immunosuppression, opportunistic infections, and/or use of antiretroviral drugs contribute to coagulopathy and gallbladder ischemia.

Indications for emergency surgery in patients who test positive for Covid-19 should not differ from those who test negative. When gangrenous cholecystitis is suspected at CT scan [7], percutaneous cholecystostomy is contraindicated. Rather, an early laparoscopic cholecystectomy may promptly reduce the inflammatory systemic response and improve the postoperative outcome even in high-risk patients recovering from Covid-19.

Luigi Bonavina

Luigi.bonavina@unimi.it

1 Department of Biomedical Sciences for Health, Division of General and Foregut Surgery, University of Milan, IRCCS Policlinico San Donato, Via Morandi 30, 20097 San Donato Milanese, Milan, Italy 

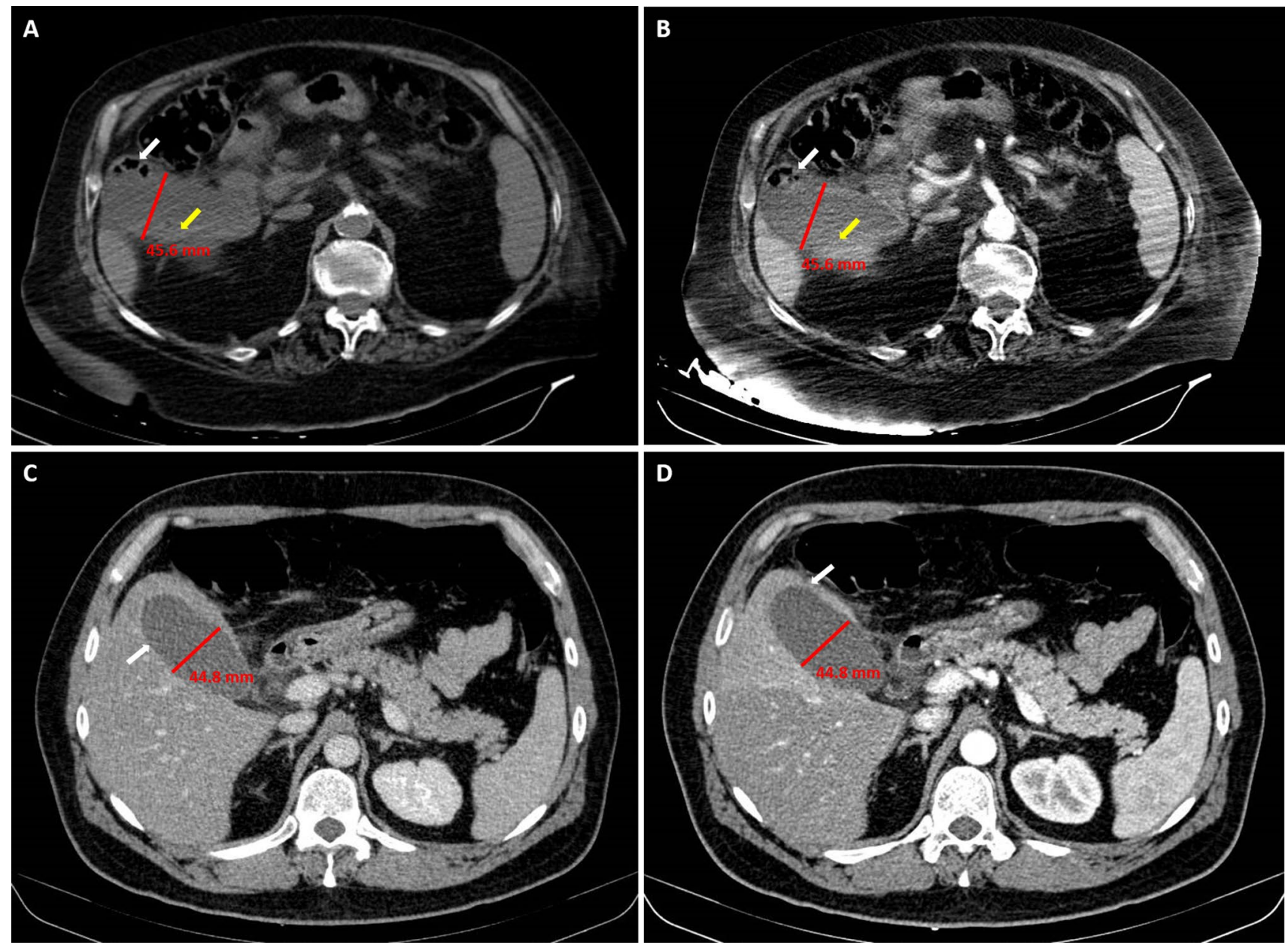

Fig. 1 Contrast-enhanced CT scans showing markedly distended and thickened gallbladder with reduced wall enhancement. a, b Without (a) and with (b) contrast enhancement (arterial phase). White arrow indicates gas in the gallbladder wall. Yellow arrow indicates intralu-

minal linear densities. c, d Without (c) and with (d) contrast enhancement (arterial phase). White arrow indicates the absence of gallbladder wall enhancement (c) and perforation of the fundus (d)

\section{References}

\section{Compliance with ethical standards}

Conflict of interest The authors declare they have no conflict of interest.

Research involving human participants and/or animals All procedures were in accordance with institutional standards and with the $1964 \mathrm{Hel}-$ sinki declaration and its later amendments.

Informed consent Written informed consent was obtained from all patients included in this study.

1. Gao Y, Xi H, Chen L (2020) Emergency surgery in suspected COVID-19 patients with acute abdomen: case series and perspectives. Ann Surg. https://doi.org/10.1097/SLA.000000000000396 1(April 13)

2. Hernandez-Rodriguez J, Tan CD, Rodriguez ER, Hoffman GS (2014) Single-organ gallbladder vasculitis. Characterization and distinction from systemic vasculitis involving the gallbladder. An analysis of 61 patients. Medicine 93:405-413

3. Guan WJ, Ni ZY, Hu Y et al (2020) Clinical characteristics of Coronavirus disease 2019 in China. N Engl J Med 382:1708-1720

4. Wu C, Chen X, Cai Y et al (2020) Risk factors associated with acute respiratory distress syndrome and death in patients with Coronavirus disease 2019 pneumonia in Wuhan, China. JAMA Intern Med. https://doi.org/10.1001/jamainternmed.2020.0994(March 13)

5. Varga Z, Flammer AJ, Steiger P et al (2020) Endothelial cell infection and endotheliitis in COVID-19. Lancet. https://doi. org/10.1016/S0140-6376(20)30917-X(April 15) 
6. Zhang Y, Xiao M, Zhang S et al (2020) Coagulopathy and antiphospholipid antibodies in patients with Covid-19. N Engl J Med. https://doi.org/10.1056/NEJMc2007575(April 8)

7. Chang WC, Sun Y, Wu EH et al (2016) CT findings for detecting the presence of gangrenous ischemia in cholecystitis. AJR 207:302-309
Publisher's Note Springer Nature remains neutral with regard to jurisdictional claims in published maps and institutional affiliations. 